\title{
Integrated Genome-Wide Pathway Association Analysis with INTERSNP
}

\author{
Christine Herold ${ }^{\mathrm{a}}$ Manuel Mattheisen ${ }^{\mathrm{b}-\mathrm{e}}$ André Lacour $^{\mathrm{a}}$ \\ Tatsiana Vaitsiakhovich $^{\mathrm{b}}$ Marina Angisch ${ }^{\mathrm{b}}$ Dmitriy Drichel $^{\mathrm{a}}$ Tim Becker ${ }^{\mathrm{a}} \mathrm{b}$ \\ a German Center for Neurodegenerative Diseases (DZNE), b Institute for Medical Biometry, Informatics and \\ Epidemiology, 'Institute of Human Genetics, Department of Genomics, Life and Brain Center, and dinstitute of

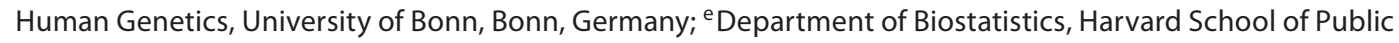 \\ Health, Boston, Mass., USA
}

\section{Key Words}

GWAS · Pathway association analysis • INTERSNP •

Parallel computing

\begin{abstract}
Objectives: Pathway association analysis (PAA) tests for an excess of moderately significant SNPs in genes from a common pathway. Methods: We present a Monte-Carlo simulation framework that allows to formulate the main ideas of existing PAA approaches using a self-contained rather than a competitive null hypothesis. A stand-alone implementation in INTERSNP makes time-consuming communication with standard GWAS software redundant. By additional parallelization with the OpenMP API, we achieve a reduction in running time for PAA by orders of magnitude, making a power simulation study for PAA feasible. Our approach properly accounts for linkage disequilibrium and is robust with respect to residual $\lambda$ inflation. Results: We demonstrate that under simple, realistic disease models, PAA can actually strongly outperform the GWAS single-marker approach. PAA methods that make use of the strength of the SNP association (GenGen, Fisher's combination test), in general, perform better than ratio-based methods (ALIGATOR, SNP ratio), whereas the relative performance of gene-based scoring (ALIGATOR, GenGen) and pathway-based scoring (SNP
\end{abstract}

ratio, Fisher's combination test) depends on the architecture of the assumed disease model. Finally, we present a new PAA score that models independent signals from the same gene in a regression framework and show that it is a reasonable compromise that combines the advantages of existing ideas.

Copyright @ 2012 S. Karger AG, Basel

\section{Introduction}

The ongoing GWAS era has led to 1,449 published association findings at $\mathrm{p}<5 \times 10^{-8}$ for 237 traits by June 2011 [1]. Typically small effect sizes leave a large portion of disease susceptibility unexplained, a phenomenon that has become famous as 'the case of the missing heritability' $[2,3]$. There is growing evidence that undetected association may partially reside in genetic variation with even lower odds ratios [4], characterized by an allelic odds ratio less than, say, 1.05. Such tiny effects may be discovered in studies with ever increasing sample size, but it is also obvious that this strategy will reach its limits when effect sizes are still smaller. When a disease is caused by hundreds or thousands of genetic variants with

\section{C.H., M.M. and A.L. contributed equally to this paper.}

\section{KARGER}

Fax +41613061234

E-Mail karger@karger.ch

www.karger.com
(C) 2012 S. Karger AG, Basel

$0001-5652 / 12 / 0732-0063 \$ 38.00 / 0$

Accessible online at:

www.karger.com/hhe
PD Dr. Tim Becker

German Center for Neurodegenerative Diseases (DZNE)

Institute for Medical Biometry, Informatics, and Epidemiology, University of Bonn

Sigmund-Freud-Str. 25, DE-53105 Bonn (Germany)

Tel. +49228287 14812, E-Mail Tim.Becker@dzne.de 
tiny effects, single-point methods will be an inappropriate tool to identify the subset of relevant SNPs from a genome-wide SNP panel. Additional information on the joint behavior of genetic variants coming from other sources could be helpful in such situations: systems biology provides 'meaningful' categories of related biological entities, such as biological pathways from databases like KEGG [5] and Biocarta [6], or Gene Ontology terms [7], which can be used to define pathway-like units [8]. The basic idea of pathway association analysis (PAA) is that disease association of a pathway may become prominent via an excess of (moderately) significant SNPs belonging to the genes of the pathway. The potential of PAA has recently been emphasized in review articles $[9,10]$ and was exemplified by the description of a pathway centered on interleukin (IL)-12 and IL-23, and the association of respective SNPs with Crohn's disease.

As pointed out by Wang et al. [9], the idea of pathwaybased analysis of GWAS data originated from the analysis of gene expression levels and respective gene set enrichment analysis (GSEA) [11]. When the underlying idea is transferred to PAA of GWAS SNP data, the main task is to develop a statistical test that judges the significance of an excess of significant SNPs in a pathway in a statistically valid manner. The latter is not trivial, because of overlap of pathways caused by genes and SNPs belonging to the same pathway and because of linkage disequilibrium (LD) between SNPs from the same gene. An early approach relied on data mining techniques [12] but has already highlighted the necessity of simulation procedures for significance assessment.

Currently, a couple of main PAA approaches exist, among them methods by Wang et al. [13], O'Dushlaine et al. [14], Holmans et al. [8], Chai et al. [15], Medina et al. [16], Elbers et al. [17], De la Cruz et al. [18], Peng et al. [19], Luo et al. [20], Nam et al. [21], Weng et al. [22], and Wang et al. [23]. All approaches have in common that they formalize a pathway as a set of genes, which is then formalized as a set of SNPs, namely the set of SNPs located within or in proximity of the gene. A PAA method can be fully characterized by 6 features: a single-marker association test, a gene scoring function, a pathway scoring function, a pathway significance testing procedure and, finally, a multiple testing adjustment procedure. Finally, two types of null hypothesis are considered [10], a 'competitive' null hypothesis: 'the genes in a gene set show the same magnitude of associations with the disease phenotype compared with genes in the rest of the genome', or the classic null hypothesis: 'no association of the pathway with phenotype'. The classic null hypothesis is also-called 'self-con- tained' null hypothesis in the context of PAA $[9,10]$. We briefly recall some major pathway association methods in terms of these features.

Wang et al. [9] were the first to introduce a method for PAA to the field of genetic epidemiology. They provided the frequently used GenGen software package (http:// www.openbioinformatics.org/gengen/). The gene score applied by this software package is defined as follows: for each gene $G$, the maximum test statistic $\operatorname{Max}(G)$ is computed. The maximum is taken over the test statistics of all SNPs that lie either within the gene or that are less than $50 \mathrm{kB}$ away from the gene. Pathway scoring is based on these gene scores and significance is evaluated with a modified Kolomogorov-Smirnov [24] test statistic. The competitive null hypothesis is considered.

The ALIGATOR software [8] scores genes as follows: a gene is considered to be significant if it contains at least one SNP whose association $\mathrm{p}$ value is lower than some pre-specified, moderate $\mathrm{p}$ value threshold. Next, for each pathway $W$, its number of significant genes $S_{P} W$ is computed. The significance of $S_{W}$ is assessed through MonteCarlo simulations that randomize the list of significant genes. Multiple testing is accounted for by a bootstrap method that accounts for dependencies of pathways caused by SNP overlap and LD. The bootstrap has to be run in addition to the Monte-Carlo procedure. We will show below that both steps can be integrated into a single randomization procedure. The competitive null hypothesis is considered.

We refer to both the GenGen and the ALIGATOR approach as 'gene-based' methods, since they use gene scores as an intermediate step to score pathways. In contrast to GenGen, ALIGATOR does not use the association strength of a SNP within a gene, but simply considers whether the single-marker $\mathrm{p}$ value is below the pre-specified cutoff. Hence, the ALIGATOR pathway score depends only on the portion of significant genes. Therefore, we call the ALIGATOR method 'ratio-based'. The characteristics of the various PAA approaches are summarized in table 1.

The SNP ratio test software [14] (http://sourceforge.net/ projects/snpratiotest/) is a 'pathway-based' method (table 1) in our terminology, since it performs no gene scoring. The pathway scoring function is the portion of 'significant' SNPs in the investigated pathway. A SNP is considered to be 'significant' if its association $p$ value is smaller than some pre-defined, moderate $p$ value cutoff. The SNP ratio test is, as the name indicates, also a 'ratiobased' method (table 1). Association $\mathrm{p}$ values for pathways are computed using Monte-Carlo simulations based on ge- 
nome-wide randomization of case-control status. A multiple testing procedure that accounts for the non-independence of different pathways is not provided. Instead, Bonferroni correction by the number of pathways has to be applied. The self-contained null hypothesis is considered.

A 'pathway-based' approach that uses the strength of association has been proposed by Peng et al. [19]. They consider the pathway test statistic $T=-2 \Sigma_{r_{i} \in W} \log \left(r_{i}\right)$ that is motivated by Fisher's combination test [25]. Here, $p_{i}$ is the p value belonging to SNP $r_{i}$ and the sum runs over all SNPs $r_{i}$ from the pathway $W$. Significance of $T$ is determined by means of Monte-Carlo simulations based on genome-wide randomization of case-control status. The self-contained null hypothesis is considered. Peng et al. [19] did not provide an implementation of the method. A method based on Fisher's combination test has also been suggested by Chai et al. [15]. These authors estimated a covariance matrix to account for LD between SNPs.

All introduced methods need simulations to judge the significance of proposed pathway scores. Some simulation schemes can only be applied to the particular score. The randomization procedure of ALIGATOR, for instance, is only applicable to gene lists that are dichotomized into a significant and non-significant category. A major goal of this paper is to show that the main ideas of the existing scoring methods can be formulated within a common Monte-Carlo simulation framework in such a way that the classic, self-contained null hypothesis can be addressed. It goes without saying that pathway methods lead to typically long running analyses. While the analysis of a single or a few real data sets is feasible within a few hours to some days with all the methods described here, it is not possible to analysis hundreds of simulated data sets with these tools required for a power comparison. For this reason, we will present an implementation of established scores such as the SNP ratio, the Gene Ratio (ALIGATOR) or Fisher-type scores. While we are able to implement several standard methods and to include them in our simulation study, it was not possible to evaluate other important approaches including GESBAP [16], GSA-SNP [21] or SSEA [22], whose implementations rely on either graphical user interface or web application.

The Monte-Carlo procedure we are going to use is based on sample permutation and allows adjustment for multiple testing with the minP approach [26]. The multiple testing adjustment does not require relevant additional running time, an opportunity that seems to have been overlooked until now. The minP approach avoids nested simulations and has previously been used in the context of haplotype analysis [27].

Pathway Association Analysis
Table 1. Overview of scoring functions

\begin{tabular}{|c|c|c|}
\hline & Ratio-based & Using strength of association \\
\hline $\begin{array}{l}\text { Gene- } \\
\text { based }\end{array}$ & $\begin{array}{l}\text { ‘Gene ratio’ } \\
\text { (ALIGATOR) }\end{array}$ & $\begin{array}{l}\text { 'best' SNP per gene } \\
\text { ('Fisher Max score'/GenGen) } \\
-2 \cdot \Sigma_{G} \log \left(p_{G}\right)\end{array}$ \\
\hline $\begin{array}{l}\text { Pathway- } \\
\text { based }\end{array}$ & $\begin{array}{l}\text { 'SNP ratio' } \\
\text { (SNP ratio test) }\end{array}$ & $\begin{array}{l}\text { all 'significant' SNPs } \\
\text { (Fisher score) } \\
-2 \cdot \Sigma \log (p)\end{array}$ \\
\hline
\end{tabular}

We provide a stand-alone implementation of the Monte-Carlo framework in our INTERSNP software, a tool originally developed for interaction analysis [28]. All components of PAA, including a single-marker analysis of replicated data sets, are handled internally such that time-consuming communication with standard GWAS software, e.g. PLINK [29], is made redundant. We will also introduce the extended pathway score 'MaxPlus' that makes use of multiple independent signals within an LD region and present results from a power simulation study.

\section{Methods}

Gene and Pathway Scores

Let $n$ be the total number of SNPs in a GWAS, let $M(G)=$ $\left\{r_{1}, r_{2}, \ldots, r_{k(G)}\right\}$ be the set of SNPs belonging to a gene $G$ and let $M(W)=\left\{r_{1}, r_{2}, \ldots, r_{k(W)}\right\}$ be the set of SNPs belonging to a pathway $W$. Let $p^{*}$ be a pre-specified $p$ value cutoff, for instance $p^{*}=0.05$. SNPs with a $\mathrm{p}$ value lower than $p^{*}$ are called 'significant' SNPs. Let $p_{i}$ be the $\mathrm{p}$ value belonging to SNP $r_{i}$. Furthermore, let $M^{*}(G)=\left\{r_{i_{1}}, r_{i_{2}}, \ldots, r_{l(G)}\right\}$ be the set of SNPs $r_{i_{j}}$ from $G$ for which $p_{i_{j}} \leq p^{*}$ and let $M^{*}(W)=\left\{r_{i_{1}}, r_{i_{2}}, \ldots, r_{l(W)}\right\}$ be the analogous set for pathways. Thus, $M^{*}(G)$ is the set of significant SNPs from $G$, and $l(G)$ is the number of significant SNPs from $G$, and $M^{*}(W)$ is the set of significant SNPs from $W$, and $l(W)$ is the number of significant SNPs from $W$. In addition, we assume that the SNPs of $M^{*}(G)$ and $M^{*}(W)$ are ordered by decreasing significance.

First, we define a gene score $A(G)$ according to $A(G)=1$, if $l(G)>0$, and $A(G)=0$ otherwise. In other words, genes are dichotomized into scores ' 1 ' and ' 0 ' according to the presence or absence of at least one significant SNP in the gene. We refer to $A(\cdot)$ as the ALIGATOR gene score, since this is the dichotomization used by the ALIGATOR software. $A(\cdot)$ can be used to define a pathway score according to

$$
A(W)=\text { number of }\{G \mid A(G)=1\} / \text { number of }\{G \mid G \in W\} .
$$

We refer to this as the 'gene ratio', since it is the number of significant genes divided by the number of genes per pathway. We note that the ALIGATOR software does not explicitly calculate the gene ratio and does not use this terminology. However, the $p$ 
value calculation of ALIGATOR is implicitly determined by this gene ratio and, therefore, we think that it is, for reason of simplification, appropriate to call $A(W)$ the 'ALIGATOR pathway score'.

In contrast to ALIGATOR, the GenGen software considers the strength of the association of the best SNP within a gene. The GenGen gene score $\operatorname{Max}(G)$ is defined as $\operatorname{Max}(G)=-2 \log \left(p_{i_{1}}\right)$, if $l(G)>0$, and $\operatorname{Max}(G)=0$ otherwise. Here, $p_{i_{1}}$ is the smallest $\mathrm{p}$ value observed for any of the SNPs from $G$. Notably, our definition of the gene score $\operatorname{Max}()$ slightly differs from that of GenGen. Our gene score is truncated, since we set $\operatorname{Max}(G)=0$, if none of the SNPs from $G$ has a $p$ value smaller than $p^{*}$. In this way, we wish to reduce the noisiness of the score in order to gain power. Note, that the choice $p^{*}=1$ yields the original GenGen gene score. Based on the gene score $\operatorname{Max}(\cdot)$, the GenGen pathway score $\operatorname{Max}(W)$ is defined as

$$
\operatorname{Max}(W)=\sum_{G \in W} \operatorname{Max}(G) .
$$

GenGen does not explicitly calculate the pathway score $\operatorname{Max}(W)$ as defined here, but judges the significance of pathways according to an enrichment of genes $G$ with high values of $\operatorname{Max}(G)$ in the pathway. The score $\operatorname{Max}(W)$ will be high in just these situations, and, therefore, can be considered as an approximation of the GenGen pathway score. Since $\operatorname{Max}(W)$ is computed from the gene score $\operatorname{Max}(G)$ with the formula from Fisher's combination test, we refer to the pathway score $\operatorname{Max}(W)$ as 'Fisher Max score'.

The next two methods, the SNP ratio test and Fisher's combination test, are pathway based (table 1) and do not use a gene score. The SNP ratio $(S R)$ is defined as

$$
S R(W)=l(W) / k(W) .
$$

In other words, $S R(\cdot)$ is the quotient of significant SNPs and the total number of SNPs in a pathway.

Next, motivated by Fisher's combination test, we define the Fisher score $F(W)$ as

$$
F(W)=-2 \sum_{r_{i_{j}} \in M^{*}(W)} \log \left(p_{i_{j}}\right) .
$$

Thus, the sum runs over all SNPs with a p value smaller than $p^{*}$. Again, this is a slight modification of a suggested score: Peng et al. [19] define $F(\cdot)$ as the sum over all SNP p values from the pathway, without truncation. The idea of truncating the score has also been suggested by De la Cruz et al. [18]. Our simulation study will show that truncation can have a considerable effect on power.

Finally, we introduce a pathway score that combines previous ideas and makes extended use of the information that is present within a gene. We have previously reviewed that the presence of multiple independently associated SNPs within one gene is a common phenomenon [30]. None of the methods described above makes proper use of independent signals that remain after adjustment for LD. While ALIGATOR and GenGen completely ignore such signals, the SNP ratio and the Fisher score increase even when associated SNPs are in perfect LD, i.e. in a situation in which the SNPs do not yield additional information. Thus, all current approaches will not have optimal power in the presence of multiple independent association signals within one gene. We define a gene score that makes use of independent signals as follows: let now $M(G)=\left\{r_{1}, r_{2}, \ldots, r_{l(G)}\right\}$ be the set of SNPs belonging to a gene $G$ and let $r_{1} \in M$ be the SNP with the best p value $p_{1}$ among the SNPs from $M$. If $p_{1}$ is smaller than the pre-specified significance threshold $p^{*}$, the remaining SNPs of the gene are tested for independent effects using logistic regression (or linear regression for quantitative trait data). We assume that a singlemarker test with 1 degree of freedom is used. Modification to the genotypic case is straightforward and part of our implementation, but will not be described any further in this paper. Let $x_{1}$ be the predictor variable that reflects the allelic effect of the best associated SNP $r_{1}$, and let $x$ be the predictor variable of the allelic effect of a further SNP $r$. Then, comparing the likelihood (L2) $\ln (p(1-p))=a+b_{1} x_{1}+b x$ against (L1) $\ln (p(1-p))=a+b_{1} x_{1}$ yields a 1-df test for the independent effect of SNP $r$. Let $r_{2} \in M-\left\{r_{1}\right\}$ be the SNP with the smallest $\mathrm{p}$ value $p=p_{2}$ after adjustment for $r_{1}$. In case $p_{2}$ is smaller than the pre-specified significance threshold, one continues with comparison of (L3) $\ln (p(1-p))=a+b_{1} x_{1}+$ $b_{2} x_{2}+b x$ against (L2) $\ln (p(1-p))=a+b_{1} x_{1}+b_{2} x_{2}$, in order to find an independent effect of SNP $r$ after adjustment for $r_{1}$ and $r_{2}$. The process continues iteratively until no further independent effects are found and results in a set $N^{*}(G)=r_{1}, r_{2}, \ldots, r_{t}$ with a corresponding set of $\mathrm{p}$ values $P^{*}(G)=\left\{p_{1}, p_{2}, \ldots, p_{t}\right\}$, where $p_{i} \leq$ $p^{*}$ and where $p_{i}$ is the $\mathrm{p}$ value for SNP $r_{i}$ after adjustment for $r_{1}$, $r_{2}, \ldots, r_{i-1}, 1 \leq i \leq t$. In case $p_{1}$ is larger than the pre-specified significance threshold, $N^{*}(G)$ is the empty set. Again motivated by Fisher's combination test, the p values are combined to form a pathway score according to

$$
F \operatorname{Max}(W)=-2 \sum_{G \in W} \sum_{j \in P^{*}(G)} \log \left(p_{j}\right) .
$$

Here, the sum runs over all genes $G$ from $W$ and all p values $p_{j}$ from $P^{*}(G)$. Again, $S(\cdot)$ is a truncated sum of non-independent $\mathrm{p}$ values and, therefore, does not follow a $\chi^{2}$ distribution. We refer to the score (in equ. 5) as 'Fisher MaxPlus'. We note that idea just described is related to a recently described approach to gene set analysis [31] that models the joint association of independent, representative eigenSNPs within a gene. In addition, multiple SNPs within one gene are also considered by Weng et al. [22] with a Kolmogorov-Smirnov-type test [24].

\section{Gene and Pathway Scores: Adjustment for Inflation Factor $\lambda$}

So far we have considered the sets $M^{*}(G)=\left\{r_{i_{1}}, r_{i_{2}}, \ldots, r_{l(G)}\right\}$ of SNPs $r_{i}$ from $G$ for which $p_{i_{j}} \leq p^{*}$, and the analogous sets $M^{*}(W)=\left\{r_{i_{1}}, r_{i_{2}}, \ldots, r_{k(W)}\right\}$ for pathways $W$. As already indicated, pathway $\mathrm{p}$ value computation will be based on genome-wide Monte-Carlo simulations (see also the following section). In case the genome-wide inflation factor $\lambda[32$ ] is greater than 1 , the Monte-Carlo procedure may be anti-conservative when the above score definitions are used, an issue that has also been raised in the recent review on pathway association methods by Wang et al. [9]. Indeed, the number $E$ of SNPs that are expected to have a p value smaller than $p^{*}$ is $E=n \cdot p^{*}$ in case $\lambda=1$, both for the real data and also for the replicated data sets. However, in case $\lambda>1$, we expect to have more than $E$ 'significant' SNPs in the real data, but not in the replicated data sets. Indeed, the permutation procedure removes residual inflation due to population stratification. For the replicated data sets, $\lambda$ always lies in the range $[0.995,1.005]$, even for real data sets with $\lambda=1.1$.

As a consequence, ratio-based scores will also be systematically inflated when there is residual inflation. In addition to that, each SNP p value will be biased, which will lead to an additional inflation of the scores that use the strength of association. In order 
to circumvent this problem, we define $M^{* *}(G)=\left\{r_{i_{1}}, r_{i_{2}}, \ldots, r_{l^{* *}(G)}\right\}$ to be the set of SNPs $r_{i_{j}}$ from $G$ for which $p_{i_{j}}$ is among the smallest $E$ p values. $M^{* *}(W)$ and $P^{* *}(G)$ can be defined analogously. Moreover, for $p_{i_{j}}$ we define the corresponding $\lambda$-adjusted $\mathrm{p}$ value $p_{i_{j}}^{\lambda}$ as follows: let $t_{i_{i}}$ be the test statistic corresponding to $p_{i}$. Then $p_{i}^{\lambda}$ is the $\mathrm{p}$ value that is computed from $t_{i_{j}} / \lambda$. With this definitions it is now possible to adjust the gene and pathway scores such that a valid Monte-Carlo procedure will be obtained even when $\lambda>$ 1. The modified scores are (1) $A(W)=$ number of $\{G \mid A(G)=1\} /$ number of $\{G \mid G \in W\}$, with $A(G)=1$, if $l^{* *}(G)>0$, (2) $\operatorname{Max}(W)=$ $\Sigma_{G \in W} \operatorname{Max}(G)$, with $\operatorname{Max}(G)=-2 \log \left(p_{i}^{\lambda}\right)$, if $l^{* *}(G)>0, \operatorname{Max}(G)=$ 0 otherwise. Furthermore, we have (3) $S R(W)=l^{* *}(W) / k(W)$, and (4) $F(W)=-2 \Sigma_{r_{i j} \in M^{* *(W)}} \log \left(p_{i_{j}}^{\lambda}\right)$. Finally, we have (5) $F$ $\operatorname{Max}(W)=-2 \Sigma_{G \in W} \Sigma_{j \in P^{* *(G)}} \log \left(p_{j}^{\lambda}\right)$.

\section{Significance Testing}

The proposed gene and pathway scores depend on gene and pathway size and the amount of LD between the SNPs from the same gene. The method of choice to account for this are MonteCarlo simulations based on genome-wide randomization of casecontrol status (quantitative trait value). Within each permutation replicate, all genotypes of an individual are kept, while case-control status is randomly permuted, resulting in a 'new' sample with a genome-wide marker panel. For each replicated data set, the pathway scores are then re-computed and compared to the score of the real data. The Monte-Carlo recipe can be summarized as follows:

(1) Compute a single-marker p value for all SNPs from the GWAS marker panel.

(2) Construct a list of the $E=n \cdot p^{*}$ best $\mathrm{p}$ values according to the user-defined significance threshold $p^{*}$.

(3) For each pathway $W$, compute one of the pathway scores (1-5).

(4) Do $N$ simulations: permute the case-control status such that the case/control ratio is kept constant or shuffle quantitative trait values of the individuals. Conduct steps 1-3 for each simulated data set.

(5) For each pathway $W$ with pathway score $S(W)$, the empirical $\mathrm{p}$ value is obtained as $s / N$, where $s$ is the number of replicated data sets and $N$ is the number of replicated data sets $k$ with a pathway score $S^{k}(W)$ greater than or equal to that of the real data.

We note that our approach is based on a permutation procedure that naturally keeps the LD pattern between the considered SNPs. Therefore, the decisive criterion for the validity of MonteCarlo simulations is met: real data and simulated data have the same properties and are 'constructed' in the same way. Thus, our method always investigates the self-contained null hypothesis 'no association between pathway and disease'. Our simulation study will prove that our method avoids potential bias caused by permutation procedures that disrupt LD between SNPs, an issue raised by Wang et al. [9].

\section{Multiple Testing Adjustment}

Multiple pathways are tested and the respective scores are not independent since genes, and consequently SNPs, may belong to more than one pathway. In addition, SNPs belonging to different pathways might be in LD. Therefore, Bonferroni correction with the number of pathways will result in a conservative procedure. The appropriate test statistic to consider is the global, self-contained null hypothesis 'none of the pathways is associated with the disease' with $T=\min P$, where $\min P$ is the smallest $\mathrm{p}$ value found for any of the pathways $W$. It would also be possible to use the maximum over all pathway scores $S(W)$ as a test statistic for the null hypothesis, but such a procedure is not reasonable, since the absolute value of $S(W)$ depends on the size of pathway $W$. Instead, consideration of $\min P$ transforms the pathway scores to an equal scale. Since the distribution of $\min P$ is, like that of the pathway scores, unknown, it has also to be evaluated with simulations. So it seems that nested simulations are necessary, since, first, the $p$ values corresponding to $S(W)$ have to be determined via simulation and, second, minP has to be evaluated by simulations, which requires Monte-Carlo(!) 'p values' for the pathway scores $S^{k}(W)$ computed in the replicated data sets $k$. Obviously, such nested simulations are computationally unfeasible. Nevertheless, it is possible to evaluate $\operatorname{minP}$ within the given Monte-Carlo framework. Ge et al. [26] have shown how the same set of permutation replicates can be used to compute both point-wise p values (in our case the $\mathrm{p}$ values per pathway) and the global $\mathrm{p}$ value that judges the significance of $\min P$. Application in the pathway setting is straightforward, since both the evaluation of $S(W)$ and $\operatorname{minP}$ use the same permutation scheme, namely genome-wide permutation of case-control status. We omit to explain the $\operatorname{minP}$ approach in more detail here, but refer to the description of the algorithm by Ge et al. [26] or to our application in the context of haplotype association analysis [27]. The validity of the $\min P$ approach has been demonstrated in these papers: re-usage of permutation replicates allows the computation of a 'global' $p$ value and yields a procedure with proper, experiment-wise $\alpha$ level.

\section{Implementation and Parallel Computing}

All methods are implemented in INTERSNP [28], a software tool for GWAS multi-marker analysis. The $\mathrm{C} / \mathrm{C}++$ source code can be downloaded from http://intersnp.meb.uni-bonn.de/. The software uses PLINK [29] genotype files as input file format. The PAA tests can be conducted with various single-marker association tests, including Armitage's trend test [33] and regression tests with covariates for case-control and quantitative trait data. Adjustment for residual inflation $(\lambda>1)$ is handled automatically. Pathway files containing the required gene and SNP information are provided for KEGG [5, 14] and Gene Ontology terms [8] constructed from the GO database [7]. We regularly accustom the KEGG release from the R-Project for Statistical Computing (KEGG.db R library, http://www.bioconductor.org/packages/2.3/data/annotation/html/KEGG.db.html) to INTERSNP file format. In this paper, we will show results for KEGG.db R accessed in May 2011 with 229 human pathways, but we will not show results for GO.

In order to speed up genome-wide Monte-Carlo simulation, we also offer a parallelized version of INTERSNP that uses the OpenMP API specification for parallel programming (http:// openmp.org/wp/). OpenMP enables simultaneous usage of processors from a common node with shared memory, and, in particular, facilitates the parallelization of loops. The respective loop cycles are distributed over the different processors. In our implementation, we parallelized the loop defining the different replicated data sets needed for the Monte-Carlo simulation procedure. For the power study described in this paper, we used 23 -fold parallelization. Computing time scaled up effectively, we achieved on average 22.9-fold speed up when we compared running time of the parallel version to that of the non-parallel version for a series of 10 selected data sets. 
Table 2. Empirical $\alpha$ levels ${ }^{\text {a }}$ for different pathway tests in the presence of $\lambda$ inflation with and without $\lambda$ adjustment

\begin{tabular}{lllll}
\hline \multirow{2}{*}{$\begin{array}{l}\text { Score } \\
\text { ment }\end{array}$} & \multirow{2}{*}{$\lambda$ adjust- $\lambda$ inflation } \\
\cline { 3 - 5 } & & & & \\
\hline Single-marker analysis & without & 0.045 & $0.083^{\mathrm{b}}$ & $0.160^{\mathrm{b}}$ \\
SNP ratio & & 0.041 & $0.088^{\mathrm{b}}$ & $0.164^{\mathrm{b}}$ \\
Fisher score & & 0.047 & $0.095^{\mathrm{b}}$ & $0.180^{\mathrm{b}}$ \\
Gene ratio & & 0.052 & 0.062 & $0.080^{\mathrm{b}}$ \\
Fisher Max & & 0.048 & 0.077 & $0.111^{\mathrm{b}}$ \\
Fisher MaxPlus & \multirow{2}{*}{ with } & 0.058 & 0.044 & 0.048 \\
SNP ratio & & 0.054 & 0.052 & 0.061 \\
Fisher score & & 0.055 & 0.048 & 0.055 \\
Gene ratio & & 0.059 & 0.044 & 0.042 \\
Fisher Max & & 0.051 & 0.050 & 0.061 \\
Fisher MaxPlus & & &
\end{tabular}

a Empirical levels are averaged over 229 KEGG pathways.

b Significant deviation from the nominal $\alpha$ level of 0.05 .

On average, the analysis of a single simulated data set took about 50 minutes at 23 -fold parallelization with $p^{*}=0.05$ and any of SNP ratio, Fisher, gene ratio and Fisher Max score. Thus, PAA will also be feasible for users that do not have access to parallel computing, running time will still be less than one day. Analysis with the Fisher MaxPlus in general took about 6 times longer than with the other scores, due to the additional regression procedure that is applied within the genes.

\section{Simulation Study}

In order to guarantee realistic LD patterns, we chose a real GWAS data set of 3,300 individuals and 500,000 SNPs (unpublished data) to serve as the basis of our simulation study. By reassignment of cases-control status according to varying disease models, we derived series of new GWAS data sets reflecting different scenarios. First, we simulated 100 data sets under the global null hypothesis 'no association' by completely random re-assignment of case-control status. In particular, those data sets were data sets under the null hypothesis 'no association at the pathway level'. Next, we produced data sets with residual inflation, since we wanted to investigate the robustness of our approach in this situation. To this purpose, we took the data sets simulated under the null hypothesis and duplicated some of the individuals, i.e. we created additional individuals with identical genome-wide genotype information as in other individuals from the sample. In that way, inflation was produced that was homogeneously distributed over the genome. Doubling 165 individuals $(n=330)$ lead to a genome-wide inflation factor of $\lambda=1.05(\lambda=1.10)$. Finally, we selected 2 KEGG pathways of medium size, hsa49300 and hsa00010, in order to simulate data sets with association at the pathway level. The first pathway consists of 50 genes, the second consisted of 55 genes. SNPs were assigned to be causal according to 3 different SNP density models: one with the number of causal SNPs proportional to the number of genes in the pathway, the second with the number of causal SNPs proportional to the total number of SNPs in the pathway, and the third with the number of causal SNPs proportional to the number of LD-pruned SNPs. Pruning was performed with PLINK [29] at an $r^{2}$ threshold of 0.50. In more detail, we defined one SNP from every second gene to be causal in scenario A, defined $10 \%$ of all SNP from the pathway to be causal in scenario B, and defined $10 \%$ of all SNP of the LD-pruned SNPs to be causal in scenario C. The causal SNPs were drawn at random once and then fixed for all simulated data sets. For each SNP, we assigned one of its alleles to be causal. In scenarios A and $B$, we chose a disease model with a fixed relative risk $r$ for all disease alleles (see below). For each individual $i$, we then computed their probability $f_{i}$ of being a case by aggregating the relative risks over the causal SNPs according to the actual allelic status of the individual at these disease SNPs. The relative risks were accumulated according to a completely additive risk model ('Fisher model'). Based on $f_{i}$, individuals were re-assigned to be either a case or a control in each simulated data set. To this purpose, a random number $z$ between 0 and 1 was drawn, and individual $i$ was assigned to be a case when $z \leq f_{i}$, and assigned to be a control otherwise. In total, 100 data sets were simulated under scenarios A and B. The actual allelic effect size $r$ has been calibrated by a pilot study as follows: we started from $r=1.2$ and reduced $r$ until the power of a single-marker analysis on the genome-wide level approached the nominal level. More precisely, we reduced $r$ in steps of 0.005 until the power of the single-marker analysis had fallen below 0.10 , based on 50 simulated data sets. This procedure resulted in $r=1.045$ for scenario A and $r=1.055$ for scenario B. For scenario C, we chose a 'two-sided threshold model': for each individual we counted the number of disease alleles and used the allele count distribution as the basis for the simulation. For individuals outside the range of two standard deviations, we chose $f_{i}=0.80$ and $f_{i}=0.0125$ for the upper and lower quantile, respectively. For individuals outside the range of one standard deviation, we chose $f_{i}=0.15$ and $f_{i}=0.066$ for the upper and lower quantile, respectively. Finally, we assumed a baseline risk of $f_{i}=0.1$ for individuals in the middle of the distribution.

Finally, all data sets were analyzed with all described pathway methods, using 5,000 permutation replicates for each data set. For each data set, we computed its global $p$ value, i.e. adjusted for the number of KEGG pathways, and computed the power as the portion of data sets with corrected $p$ values less than or equal to 0.05 . Genome-wide single-marker $\mathrm{p}$ values were also determined with genome-wide Monte-Carlo simulations.

\section{Results}

Results obtained under the null hypothesis can be found in table 2. In this table, we present unadjusted $p$ values at the 0.05 level. The results we report are averages taken over all pathways. Pathway-specific phenomena did not occur. When there is no residual inflation $(\lambda=$ 1.00), the single-marker analysis and all pathway methods show empirical $\alpha$ levels that do not significantly deviate from the nominal level (table 2). As expected, the single-marker analysis does not keep the nominal level when 
Table 3. Power values for different PAA scores and disease models

\begin{tabular}{|c|c|c|c|c|c|c|c|c|c|}
\hline Score & \multicolumn{3}{|l|}{ Scenario A } & \multicolumn{3}{|l|}{ Scenario B } & \multicolumn{3}{|l|}{ Scenario C } \\
\hline SNP ratio & 0.08 & 0.06 & n.d. ${ }^{c}$ & 0.51 & 0.53 & n.d. ${ }^{c}$ & 0.52 & 0.42 & n.d. ${ }^{c}$ \\
\hline Fisher score & 0.14 & 0.11 & 0.15 & 0.61 & 0.87 & 0.68 & 0.57 & 0.65 & 0.56 \\
\hline Gene ratio & 0.41 & 0.10 & n.d. ${ }^{\mathrm{c}}$ & 0.03 & 0.01 & n.d. ${ }^{c}$ & 0.15 & 0.07 & n.d. ${ }^{c}$ \\
\hline
\end{tabular}

there is residual inflation (table 2). Moreover, the pathway methods exceed the nominal level when no $\lambda$ adjustment is performed. With $\lambda=1.05$, we observe the strongest excess with the Fisher score (0.095). This is plausible since the Fisher score is biased in 2 ways, first, since too many SNPs reach the $p$ value cutoff and, second, since the strength of association is, on average, overestimated for the individual SNPs. Consistently, the SNP ratio (0.088), which is biased only by too many SNPs reaching the cutoff, and Fisher Max (0.077), which is predominantly biased by the inflation of the test statistic of the most significant SNP per gene, show less pronounced excess of the nominal level. With even stronger inflation $(\lambda=1.10)$, the phenomenon is aggravated with the most prominent excess of the nominal level again for the Fisher score (0.180). Thus, our simulations show that pathway tests can seriously be affected by residual inflation, highlighting the importance of proper adjustment. We wish to emphasize that the original implementation of the SNP ratio test [14] properly handles residual $\lambda$ inflation, and that ALIGATOR [8] and GenGen [13] also should not be affected by the problem since they use different simulation schemes. When $\lambda$ adjustment of the pathway scores is performed as described in the Methods section, all pathway tests keep the nominal level (table 2). In conclusion, we can state that the simulations under the null hypothesis demonstrate the validity of our approach and its robustness towards residual inflation.

The simulations were also used to determine the 'effective number' of pathways in the KEGG database. By comparison of Bonferroni-corrected $p$ values and the respective $\min P$-adjusted global $\mathrm{p}$ values, we computed that the appropriate correction factor was on average 160. In other words, the 229 KEGG pathways correspond to approximately 160 independent tests, reflecting overlap and LD between the genes of the pathways. It can also be stated that for a KEGG pathway a nominal $p$ value of $0.05 / 160=0.0003$ is needed to guarantee experimentwise significance at $\alpha=0.05$.

Table 3 contains power values for the single-marker analysis and all introduced pathway scores, for scenarios $\mathrm{A}$ and $\mathrm{B}$ and for 3 inclusion thresholds $p^{*}$. Power is always given for an $\alpha$ level of 0.05 , after adjustment for multiple testing. Under all scenarios, power of the single-marker analysis does not exceed the nominal level. In other words, the standard GWAS analysis approach will fail under the disease models considered here. In contrast to this, under both disease models there is at least one score that yields substantial power for PAA.

In scenario A, one causal SNP from every second gene, the Fisher Max score (motivated by GenGen) has 86\% power with inclusion threshold $p^{*}=0.05$. Power remains nearly unchanged when Fisher MaxPlus is used instead. This is not surprising since no independent effects were modeled under scenario A. The other scores perform markedly worse, only the gene ratio (motivated by ALIGATOR) has medium power (41\%). When $p^{*}=0.20$ is chosen to define 'significant' SNPs, the power values remain virtually unchanged, except for the gene ratio, for which power drops sharply to $10 \%$. Again, this is not too surprising: because of the very moderate value of $p^{*}$, almost every gene $G$ will have gene score $A(G)=1$, which makes the gene ratio unspecific and reduces the power of the test. Without truncation $\left(p^{*}=1\right)$ power of Fisher and Fisher Max improve slightly. An explanation for that is that $p^{*}<1$ leads to a loss of signals and loss of power. 
In scenario B, every 10th SNP from the pathway is causal, and for $p^{*}=0.05$ the Fisher score performs best (61\%). The Fisher Max score performs significantly worse (17\% power). An improvement to $29 \%$ is achieved with the Fisher MaxPlus score, which is consistent with the simulation setup. Although independent effects were not explicitly simulated, choosing every 10th SNP to be causal will automatically lead to independent effects in not too small genes. While the gene ratio has virtually no power under scenario $\mathrm{B}$, the SNP ratio performs second best ( $51 \%$ power) and markedly better than the Fisher Max score. In contrast to scenario A, power values depend on the inclusion criterion $p^{*}$. When $p^{*}=0.20$ is chosen to define 'significant' SNPs, the Fisher score is still the most powerful, now with excellent $89 \%$ power. The power of the other scores also improves, while the relative performance of the other scores remains unchanged. Notably, the Fisher MaxPlus performs better than the Fisher Max by 10 percentage points, which is consistent with the design of scenario $\mathrm{B}$.

In scenario $\mathrm{C}$, the Fisher score performs best again, followed by the Fisher MaxPlus score. This is plausible, since the setup leads to several causal SNPs per gene. Thus, gene-based scoring with the Fisher Max is less favorable. The choice of $p^{*}$ again has a measurable effect especially on the Fisher score. With $p^{*}=0.20$, its power improves to $65 \%$.

Taken together, it can be stated that PAA can be a powerful tool for statistical analysis of GWAS data with no evidence for association at the single-marker level. PAA methods that use the strength of association in general perform better than ratio-based methods. Indeed, the Fisher score always has better power than the SNP ratio, and the Fisher Max score always outperforms the gene ratio (table 3). In the presence of independent variants, power is improved by replacing the Fisher Max score with the Fisher MaxPlus score. The relative performance of the gene-based and pathway-based methods depends on the assumed disease model. Under scenario A, the genebased Fisher Max score was most powerful, which is consistent with the disease model, since under scenario A causal variants are assigned gene-wise. Under scenarios $\mathrm{B}$ and $\mathrm{C}$, the pathway-based Fisher score was most powerful, which again is in concordance with the setup. Since the architecture of real diseases with respect to the distribution of causal variants over genes and pathways is unknown, we recommend to use both the Fisher score and the Fisher Max score motivated by GenGen [13], or its extension Fisher MaxPlus, in real data analysis. In addition, the Fisher score should be operated with varying cutoff $p^{*}$. For the Fisher Max score, the cutoff seems to be less relevant. Thus, $p^{*}=1$ should be chosen with Fisher Max. In any case, additional multiple testing adjustment for a number of different tests and parameter choices will be obligatory in any data analysis.

Since the differences in power between various approaches are quite substantial, we believe that our conclusions are approvable even though only a limited number of simulated data sets could be investigated under each scenario. Additionally, even though power values for the original ALIGATOR and GenGen implementations will to some extent differ from the values shown here, the qualitative conclusions we could make are reliable.

\section{Discussion}

We have presented a fast and robust PAA implementation that simplifies PAA and makes its application to large GWAS data sets computationally feasible. The presented framework integrates the workflow into a single software tool and allows a flexible definition of the gene and pathway scores to be used. In particular, usage of the SNP association strength and of multiple independent effects with LD regions is possible. Existing ideas could successfully be transferred to the investigation of a selfcontained rather than a competitive null hypothesis. Although validity and strict significance assessment are the main goals of our approach, we note that exploratory tools can be used in a first analysis. In particular, methods that use a collection of different databases, such as GESBAP [16], are recommendable for an exploratory strategy.

The speed-up achieved by stand-alone implementation and parallelization rendered a power simulation study feasible. To the best of our knowledge, we present the first simulation study that makes an explicit comparison to a single-marker analysis on a genome-wide scale. With this study we could demonstrate that PAA can actually be a powerful GWAS tool when the single-marker approach fails. Thus, PAA is a useful complementary analysis strategy.

Although the PAA methods that directly use the strength of SNP association are more powerful, we wish to emphasize that methods like the SNP ratio test [14] are valuable supplements since they are more specific. Indeed, major gene effects may produce significant results for all pathways that contain the major gene, even if there is no additional evidence for association in other genes 
from such a pathway. This undesired effect may be avoided by removing major genes from the analysis [9] or by using ratio-based methods (SNP ratio, ALIGATOR) which are more robust with respect to single gene effects by design. However, the HLA region may even render the SNP ratio unspecific when autoimmune disorders are analyzed. In this case, removal of HLA from the pathway analysis seems to be the only solution [9].

We have also seen that the choice of inclusion threshold $p^{*}$ that defines single-marker significance can have a strong effect on power. For a real data set, this effect has previously been reported [8]: for Crohn's disease strong evidence was found with a strict inclusion criterion $\left(p^{*}=\right.$ 0.001 ), while for bipolar disorder significant results were also seen with $p^{*}=0.05$. For practical purposes this implies that various inclusion thresholds should be tried, of course, with the need to apply additional, conservative Bonferroni correction with the number of cutoffs tested. In view of this, the determination of the optimal $p^{*}$ from the data, together with a corresponding adjustment procedure to judge significance would be an obvious future enhancement.

We have shown that under rather simple disease models PAA may have excellent power while a single-marker analysis will fail. Consequently, PAA may be a powerful tool for unraveling parts of the missing heritability of complex human diseases. This, of course, will only be possible with a high quality of the pathway information that is plugged into the analysis. The community has to be aware of the fact that existing pathway databases are incomplete and may contain errors. However, respective knowledge is evolving, and results from PAA could give valuable feedback to improve knowledge of human pathways. In this sense, we hope that our PAA approach can contribute to the progress of pathway analysis and will also benefit from improvement of pathway databases in the coming years.

\section{URLs}

INTERSNP: http://intersnp.meb.uni-bonn.de/

GenGen: http://www.openbioinformatics.org/gengen/

SNP ratio test: http://sourceforge.net/projects/snpratiotest/

The R Project for Statistical Computing: http://www.r-project.org/ Bioconductor KEGG.db R library: http://www.bioconductor.org/ packages/2.3/data/annotation/html/KEGG.db.html

The OpenMP API specification for parallel programming: http://openmp.org/wp

\section{Acknowledgement}

The work was supported by grants BE 3828/3-2 and BE 3828/41 from the Deutsche Forschungsgemeinschaft.

\section{References}

1 Hindorff LA, Junkins HA, Hall PN, Mehta JP, Manolio TA: A Catalog of Published Genome-Wide Association Studies. Available at: www.genome.gov/gwastudies. Accessed in June 2011.

12 Maher B: Personal genomes: the case of the missing heritability. Nature 2008;456:18-21.

- 3 Manolio TA, Collins FS, Cox NJ, Goldstein DB, Hindorff LA, Hunter DJ, McCarthy MI, Ramos EM, Cardon LR, Chakravarti A, et al: Finding the missing heritability of complex diseases. Nature 2009;461:747-753.

4 Yang J, Benyamin B, McEvoy BP, Gordo, S, Hendres AK, Nyholt DR, Madden PA, Heath AC, Martin NG, Montgomerey GW, et al: Common SNPs explain a large proportion of the heritability for human height. Nat Genet 2010;462:565-571.

5 Kanehisa M, Goto S, Furumichi M, Tanabe M, Hirakawa M: KEGG for representation and analysis of molecular networks involving diseases and drugs. Nucleic Acids Res 2010;38:D355-D360.
6 Biocarta Database. Available at: http://www. biocarta.com/

7 Harris MA, Clark J, Ireland A, Lomax J, Ashburner M, Foulger R, Eilbeck K, Lewis S, Marshall B, Mungal C, et al: The Gene Ontology (GO) database and informatics resource. Nucleic Acids Res Database 2004;32:D258D261.

-8 Holmans P, Green EK, Pahwa JS, Ferreira MA, Purcell SM, Sklar P, Wellcome Trust Case-Control Consortium, Owen MJ, O'Donovan MC, Craddock N: Gene ontology analysis of GWAS study data sets provides insights into the biology of bipolar disorder. Am J Hum Genet 2009;85:13-24.

-9 Wang K, Li M, Hakonarson, H: Analysing biological pathways in genome-wide association studies. Nat Rev Genet 2010;11:843854.

10 Wang L, Jia P, Wolfinger RD, Chen X, Zhao $\mathrm{Z}$ : Gene set analysis of genome-wide association studies: methodological issues and perspectives. Genomics 2011;98:1-8.
Subramanian A, Tamayo P, Mootha VK, Mukherjee S, Ebert BL, Gillette MA, Paulovich A, Pomeroy SL, Golub TR, Lander ES, et al: Gene set enrichment analysis: a knowledge-based approach for interpreting genome-wide expression profiles. Proc Natl Acad Sci USA 2005;102:15545-15550.

12 Dinu V, Zhao H, Miller PL: Integrating domain knowledge with statistical and data mining methods for high-density genomic SNP disease association analysis. J Biomed Inform 2007;40:750-760.

13 Wang K, Li M, Bucan M: Pathway-based approaches for analysis of genome-wide association studies. Am J Hum Genet 2007;81: 1278-1283.

14 O’Dushlaine C, Kenny E, Hero, EA, Segurado R, Gill M, Morris DW, Corvin A: The SNP ratio test: pathway analysis of genomewide association datasets. Bioinformatics 2009;25:2762-2763. 
15 Chai HS, Sicotte H, Bailey KR, Turner ST, Asmann YW, Kocher JP: GLOSSI: a method to assess the association of genetic loci-sets with complex diseases. BMC Bioinformatics 2009;10:102.

16 Medina I, Montaner D, Bonifaci N, Pujana MA, Carbonell J, Tarraga J, Al-Shahrour F, Dopazo J: Gene set-based analysis of polymorphisms: finding pathways or biological processes associated to traits in genomewide association studies. Nucleic Acids Res 2009;37:340-344.

17 Elbers CC, van Eij KR, Franke L, Mulder F, van der Schouw YT, Wijmenga C, OnlandMoret NC: Using genome-wide pathway analysis to unravel the etiology of complex diseases. Genet Epidemiol 2010;33:419-431.

-18 De la Cruz O, Wen X, Ke B, Song M, Nicolae DL: Gene, region and pathway level analyses in whole-genome studies. Genet Epidemiol 2010;34:222-231.

19 Peng G, Luo L, Si H, Zhu Y, Hu P, Hong S, Zhao J, Zhou X, Reveille JD, Amos CI, et al: Gene and pathway-based second-wave analysis of genome-wide association studies. Eur J Hum Genet 2010;18:111-117.
20 Luo L, Peng G, Zhu Y, Dong H, Amos CI, Xiong M: Genome-wide gene and pathway analysis. Eur J Hum Genet 2010;18:111-117.

21 Nam D, Kim J, Kim SY, Kim S: GSA-SNP: a general approach for gene set analysis of polymorphisms. Nucleic Acids Res 2010; 38:W749-W754.

22 Weng L, Macciardi F, Subramanian A, Guffanti G, Potkin SG, Yu Z, Xie X: SNP-based pathway enrichment analysis for genomewide association studies. BMC Bioinformatics 2011;12:99.

23 Wang L, Jia P, Wolfinger RD, Chen X, Grayson BL, Aune TM, Zhao Z: An efficient hierarchical generalized linear mixed model for pathway analysis of genome-wide association studies. Bioinformatics 2011;27:686692.

24 Hollander M, Wolfe DA: Nonparametric Statistical Methods. New York: Wiley, 1999.

25 Fisher RA: Statistical Methods for Research Workers, ed 4. London: Oliver and Boyd, 1932.

26 Ge Y, Dudoit S, Speed TP: Resampling-based multiple testing for microarray data analysis. Test 2003;12:1-77.

27 Becker T, Knapp M: A powerful strategy to account for multiple testing in the context of haplotype analysis. Am J Hum Genet 2004; 75:561-570.
28 Herold C, Steffens M, Brockschmidt FF, Baur MP, Becker T: INTERSNP: genomewide interaction analysis guided by a priori information. Bioinformatics 2009;25:32753281.

29 Purcell S, Neale B, Todd-Brown K, Thomas L, Ferreira MA, Bender D, Maller J, Sklar P, de Bakker PI, Daly MJ, et al: PLINK: a tool set for whole-genome association and population-based linkage analyses. Am J Hum Genet 2007;81:559-575.

30 Becker T, Herold C: Joint analysis of tightly linked SNPs in screening step of genomewide association studies leads to increased power. Eur J Hum Genet 2009; 17:1043-1049.

31 Chen LS, Hutter CM, Potter JD, Liu Y, Prentice RL, Peters U, Hsu L: Insights into colon cancer etiology via a regularized approach to gene set analysis of GWAS data. Am J Hum Genet 2010;86:860-871.

- 32 Devlin B, Bacanu SA, Roeder K: Genomic control to the extreme. Nat Genet 2004;36: 1129-1130.

33 Armitage P: Tests for linear trends in proportions and frequencies. Biometrics 1955; 11:375-386. 